\title{
A TIME-FREQUENCY APPROACH TO BLIND DECONVOLUTION IN MULTIPATH UNDERWATER CHANNELS
}

\author{
N. Martins, S. Jesus* \\ SiPLAB-FCT, University of Algarve \\ Campus de Gambelas, 8000 Faro, Portugal \\ nmartins@ualg.pt, sjesus@ualg.pt
}

\begin{abstract}
Blind deconvolution is presented in the underwater acoustic channel context, by time-frequency processing. The acoustic propagation environment was modelled as a multipath propagation channel. For noiseless simulated data, source signature estimation was performed by a model-based method. The channel estimate was obtained via a time-frequency formulation of the conventional matched-filter. Simulations used a ray-tracing physical model, initiated with at-sea recorded environmental data, in order to produce realistic underwater channel conditions. The quality of the estimates was 0.793 for the source signal, and close to 1 for the resolved amplitudes and time-delays of the impulse response. Time-frequency processing has proved to overcome the typical ill-conditioning of single sensor deterministic deconvolution techniques.
\end{abstract}

\section{INTRODUCTION}

Two fundamental problems arising in many signal processing applications are channel and source signature estimation, often referred as deconvolution. Areas such as data transmission, reverberation cancellation, seismic signal processing, image restoration, etc., are some examples where deconvolution finds application. One of these areas is ocean acoustics, where a fundamental problem is the passive characterization of acoustic transients and the ocean environment. This paper aims to give an approach to solve the problem of blind deconvolution in ocean acoustics, i.e., obtaining at once estimates of the emitted signal and the medium impulse response (IR) representative of its physical properties. In view of the typical structure of underwater channel model-IRs, constituted by a set of close arrivals, time-frequency (TF) distributions (TFDs) have been chosen as a mean for separating the various replicas of the typically transient source signal. A shallow-water realistic scenario was chosen to support the presentation.

${ }^{*}$ Thanks to MCT, FCT, PRAXIS XXI (BM/19298/99) for funding, and to Profs. A. Quinquis and C. Gervaise for the favourable reception at ENSIETA.

\author{
C. Gervaise, A. Quinquis \\ EIA Dpt., ENSIETA \\ 29806 Brest Cedex 09, France \\ gervaice@ensieta.fr, quinquis@ensieta.fr
}

2. MATHEMATICAL BACKGROUND

One of the main mathematical tools essential in the present work is the Wigner-Ville distribution (WV), defined by[1]

$$
W V_{x}(t, f)=\int_{-\infty}^{\infty} x\left(t+\frac{\tau}{2}\right) x^{*}\left(t-\frac{\tau}{2}\right) \mathrm{e}^{-\mathrm{j} 2 \pi f \tau} \mathrm{d} \tau,
$$

where * designates complex conjugate. An also important TFD is the signal-dependent distribution radially Gaussian kernel distribution (RGK)[2]

$$
R G K_{x}(t, f)=\operatorname{IFT} 2\left[\Phi_{R G K, x}(\nu, \tau) A F_{x}(\nu, \tau)\right],
$$

where $\Phi_{R G K, x}(t, f)$, the kernel of the distribution, is adapted to the signal to be analyzed, $A F_{x}(\nu, \tau)$ stands for the ambiguity function of $x(t)$, and IFT2[.] designates the bi-dimensional (2D) inverse Fourier transform operator. Signal reconstruction was performed via an inverse TF transformation. The basis method[3] was employed, where a linear combination of the basis functions $e_{k}(t)$ was used to obtain the final source signal estimate such as

$$
\hat{s}(t)=\sum_{k=1}^{N_{b}} \alpha_{k} e_{k}(t) .
$$

The functions $e_{k}(t)$ span the signal space to which the estimate is confined. Channel estimation was performed taking into account the TF formulation of the matched-filter (MF). The fact that the WV obeys to Moyal's formula[4] conduces to the following equation:

$$
\begin{gathered}
\int_{-\infty}^{\infty} \int_{x_{1}} W V_{x_{1}}(t, f) W V_{x_{2}(t+\tau), x_{2}(t)}^{*}(t, f) \mathrm{d} t \mathrm{~d} f= \\
{\left[\int_{-\infty}^{\infty} x_{1}(t) x_{2}^{*}(t+\tau) \mathrm{d} t\right]\left[\int_{-\infty}^{\infty} x_{1}(t) x_{2}^{*}(t) \mathrm{d} t\right]^{*} .}
\end{gathered}
$$

This shows that temporal correlation can be performed in the TF domain. 


\section{SIMULATION RESULTS}

The simulated data set corresponds to a canonical two-layered shallow-water waveguide whose environment is the real data acquisition scenario of the INTIMATE ' 96 sea trial[5]. The acoustic source is positioned at $90-\mathrm{m}$ depth, and the receiver is located at $5.6-\mathrm{km}$ range and $115-\mathrm{m}$ depth, as illustrated in Fig. 1. The 135-m water column is superim-

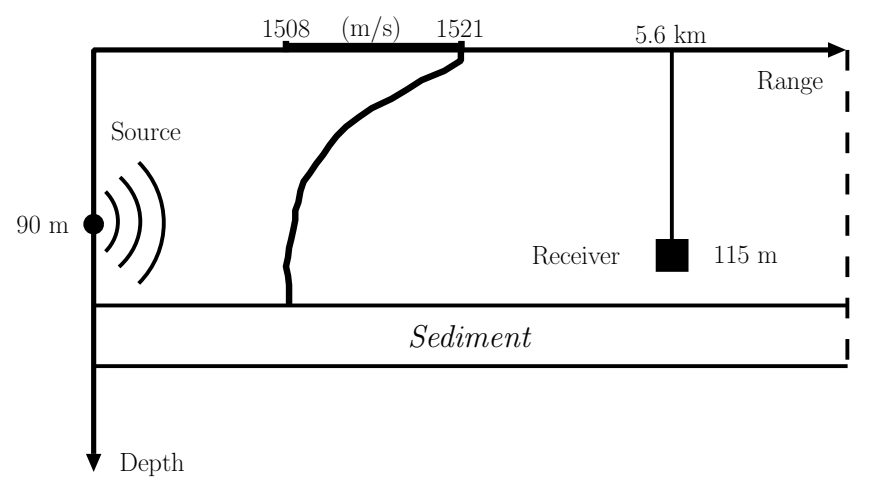

Fig. 1. Scenario of the INTIMATE '96 sea trial.

posed to a perfectly rigid-modelled bottom. The considered sound speed profile is shown within Fig. 1. Application of the ray/beam propagation model BELLHOP[6], taking as input the environment in Fig. 1, allowed to obtain the reference channel IR $h(t)-$ Fig. 2. A total number of $M=45$

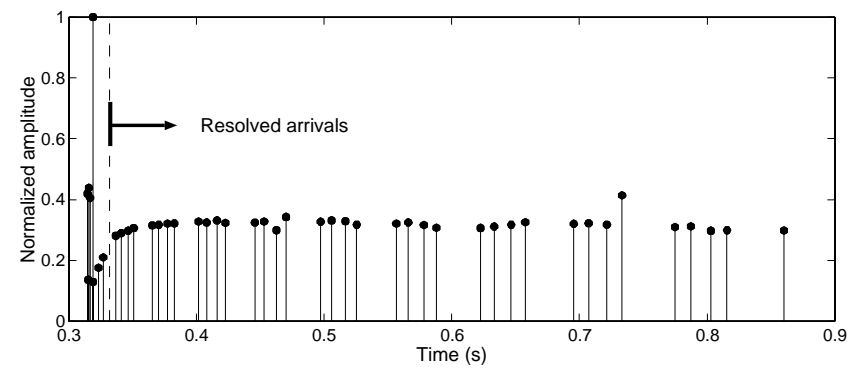

Fig. 2. Reference IR $h(t)$ of the canonical scenario. The dashed line separates unresolved from resolved arrivals.

arrivals have been predicted by the model, spanning the time interval $[0.31,0.86] \mathrm{s}$. The amplitudes $a_{m}$ and time-delays $\tau_{m}$ of the arrivals are the channel parameters to estimate, grouped into the vectors $\mathbf{a}$ and $\tau$, respectively. The source signal $s(t)$ consists of a $\mathcal{T}=0.0625$-s duration linear frequency modulation (LFM) pulse, spanning the [300, 800]$\mathrm{Hz}$ instantaneous frequency (IF) range. The IR is split into 2 packets of arrivals: one packet contains the first 8 arrivals, and the other contains the remaining 37 arrivals, which can be seen as unresolved and resolved arrivals, respectively. Unresolved arrivals are adjoint arrivals whose arrival times differ by less than the inverse of the bandwidth of $s(t)$. The received signal is modelled by

$$
r(t)=x(t)+\xi(t),
$$

where $x(t)$ and $\xi(t)$ are the signal and noise terms, respectively. Next two sub-sections describe results for noiseless data, whereas Sec. 4 addresses deconvolution robustness to noise.

\subsection{Source Signature Estimation}

Considering the multi-component structure of the noiseless received signal, source signature estimation was performed by application of the basis method, whose output is given by (3), and whose model function was defined by the product of $W V_{r}(t, f)$ by a 2D function, centered on the IF estimate of $s(t)$. This procedure is an approach to solve a multi-component signal separation problem, conditioned on the overlap between the very early arrivals.

The information of the purely phase modulated emitted signal $s(t)=\exp \left[\mathrm{j} \varphi_{i}(t)\right]$ is contained in the $\mathrm{IF} f_{i}(t)$, apart from a phase term, since the signal's amplitude modulation component is constant. This implies that most part of the signal energy concentrates around the line defined by the points $\left[t, f_{i}(t)\right]$, in the TF space[7]. This characteristic motivated source signal estimation, by a first estimation of the IF of $s(t)$. Due to the typical presence of one or a few strong arrivals in $h(t)$, the IF estimation consisted of the global maximization with respect to $t$, of the signal-dependent distribution RGK of the received signal, $R G K_{r}(t, f)$ :

$$
\left[t, \hat{f}_{i}(t)\right]=\left\{(t, f): t=\arg \max _{t} R G K_{r}(t, f), f \in \mathcal{B}\right\} .
$$

In $R G K_{r}(t, f)$, for an unitary kernel volume, the first 8 arrivals are grouped in a support of large energy, as shown in Fig. 3. The remaining 37 arrivals, "clustered" in quadruplets,

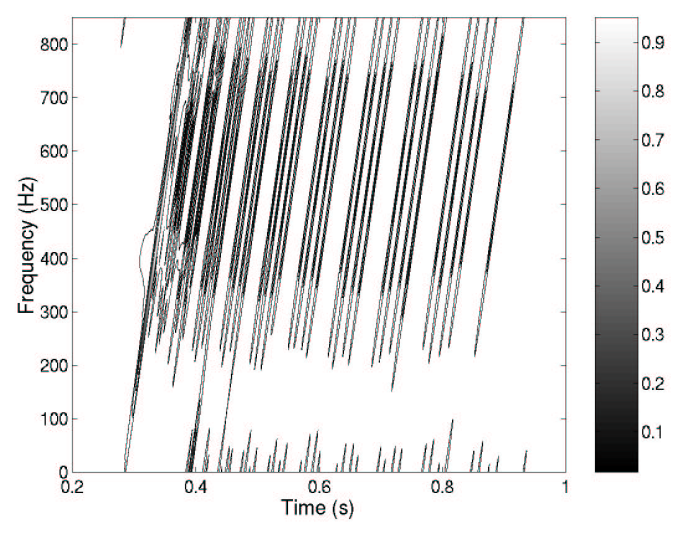

Fig. 3. Contour plot of the RGK signal-dependent distribution of the received signal, $R G K_{r}(t, f)$.

are clearly distinguished. Maximization of $R G K_{r}(t, f)$ 
with respect to $t$, gave rise to an accurate IF estimate[8]. The IF is estimated via maximization of a signal-dependent TFD, since here, the interference terms (ITs) are quite reduced, comparatively to non-signal-dependent TFDs, as the WV. In that sense, non-signal-dependent TFDs are inapplicable in (this) case of signals composed by a sum of replicas, besides the large variance of the estimate, in presence of noise.

It is likely that the proposed approach will yield a good IF estimate mainly for the class of purely phase modulated source signals, for which $W V_{s}(t, f)$ is usually concentrated on a continuous region centered on the line defined by the pair $\left[t, f_{i}(t)\right]$. However, for a non-linear frequency modulation source signal, there are always ITs in its auto-WV, what may require a smaller value for the kernel volume of $R G K_{r}(t, f)$, if some ITs of that auto-WV have greater amplitude than the signal terms. A quadratic IF has been accurately estimated in [9]. The extension to the case of inconstant amplitude modulation may hypothetically be treated in the same manner, provided that the instantaneous amplitude is a sufficiently smooth function, what weakly increases the spread of the auto-WV along the IF.

For application of the basis method, the considered signal space was the subspace of band-limited signals, in the band $\left[0, f_{s} / 2\right]$, which is a natural choice when dealing with discrete-time signals. The obtained source estimate is depicted in Fig. 4, and corresponds to a cross-correlation coefficient of 0.793 with $s(t)$. The band limitation signal cons-

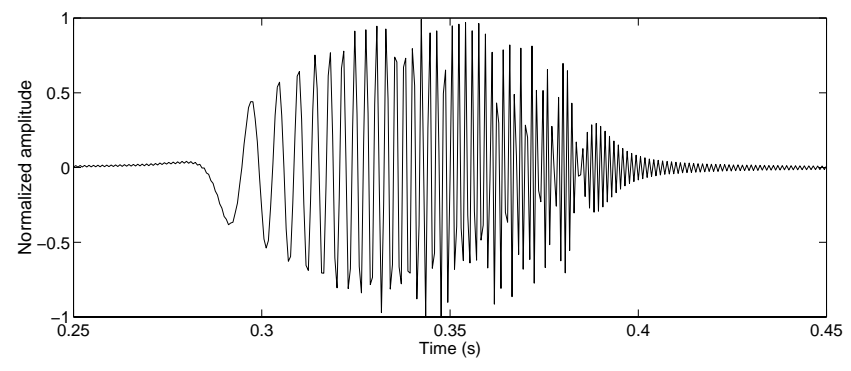

Fig. 4. Source estimate -real part of $\hat{s}(t)$.

traint is reflected not only in the increased signal duration, but also in an increase of the IF range, since the limitation to $\left[0, f_{s} / 2\right]$ gave the freedom to the estimate's IF to extend in the whole $[0,850] \mathrm{Hz}$ interval.

\subsection{Channel Estimation}

This sub-section describes the estimation of the parameters $\left\{a_{m}, \tau_{m} ; m=1, \ldots, M\right\}$ that characterize the channel's IR, by use of the TF formulation of the MF mentioned in Sec. 2. For the case of an LFM signal at emission, and for the particular cases $x_{1}(t)=r(t)$ and $x_{2}(t)=s(t)$, (4) is well approximated by the integration of $W V_{r}(t, f)$ along $f_{i}(t)$ [8]. Thus, the IF estimate obtained in Sec. 3.1 was used to do this "coherent integration" of $W V_{r}(t, f)$. The obtained channel estimate is shown in Fig. 5. For the problem at

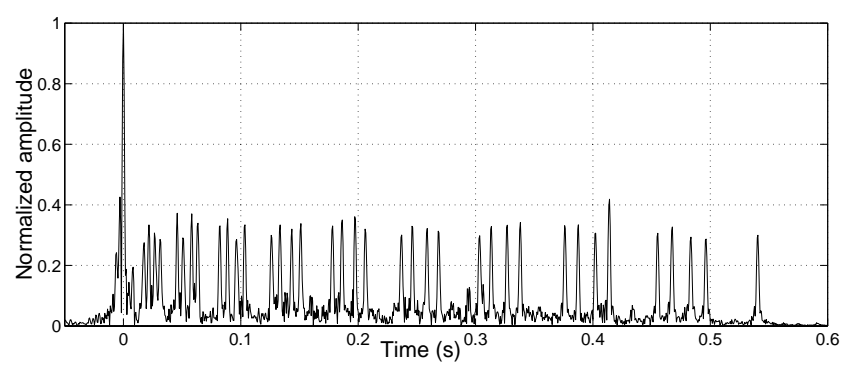

Fig. 5. Channel estimate, obtained by coherent integration of $W V_{r}(t, f)$.

hand, it can be verified that the obtained resolution is comparable to that of the MF result-Fig. 6. It can be shown

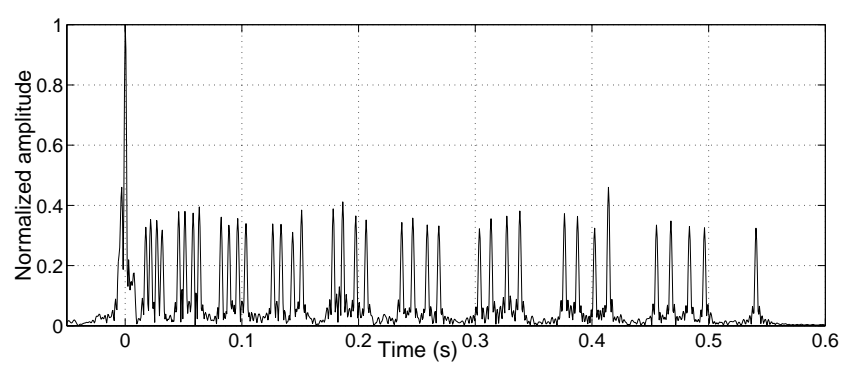

Fig. 6. Channel estimate obtained by classic matched-filtering.

that the relation between the two resolutions depends on the relative values of the IF range and the inverse of $\mathcal{T}$ [8]. In practice, the first 8 arrivals of $h(t)$ are not resolved, and the quality of the channel estimate will refer only to the remaining 37 arrivals. One normalized measure of the quality can be given as

$$
\rho_{a}=1-\frac{\|\mathbf{a}-\hat{\mathbf{a}}\|}{2} ; \quad \rho_{\tau}=1-\frac{\|\boldsymbol{\tau}-\hat{\boldsymbol{\tau}}\|}{2},
$$

where the vectors must first be unitarily normalized. The quality of the channel estimate is given by $\rho_{a}=0.9664$ and $\rho_{\tau}=0.9996$. The present approach can be applied also to $R G K_{r}(t, f)$.

Alternatively to the procedure of TF coherent integration, the channel estimate can be obtained by cross-correlation between $\hat{s}(t)$ and $r(t)$, once $\hat{s}(t)$ be available. This approach allowed to obtain an estimate very similar to the previous estimates[8].

\section{PERFORMANCE IN NOISE}

In this section, the received signal in (5) contains non-zero white noise $\xi(t)$. Looking at the expected value of 
$W V_{r}(t, f)$, one finds that

$$
\mathrm{E}\left[W V_{r}(t, f)\right]=W V_{x}(t, f)+\sigma_{\xi}^{2},
$$

where $\sigma_{\xi}^{2}$ is the noise variance. It can readily be seen that the blind deconvolution method can be performed the same way as in the noiseless data case of Sec. 3, provided that $W V_{r}(t, f)$ is replaced by its expected value. Regarding $R G K_{r}(t, f)$, one can verify that

$$
\mathrm{E}\left[A F_{r}(\nu, \tau)\right]=A F_{x}(\nu, \tau)+\sigma_{\xi}^{2} \delta(\nu) \delta(\tau),
$$

hence not representing a significant change on the calculus of the optimal kernel, relatively to the noiseless case. The signal-to-noise ratio (SNR) is defined as:

$$
\mathrm{SNR}_{\mathrm{dB}}=10 \log \frac{\frac{1}{N} \sum_{n=0}^{N-1} s^{2}(n)}{\sigma_{\xi}^{2}},
$$

where $N$ is the number of time samples. The empirical measures below were estimated from 100 trials for each SNR value. Source estimation performance in noise is illustrated in Fig. 7, by a plot of the normalized correlation coefficient as a function of the SNR. Channel estimation performance

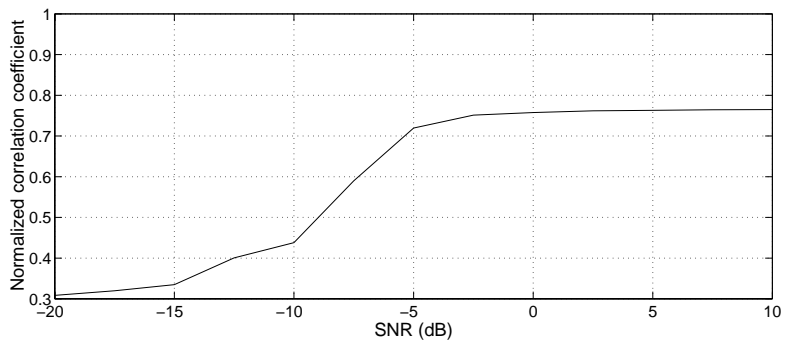

Fig. 7. Source estimation performance in noise.

in noise was measured by the normalized performance measures (7), for values of SNR ranging from -5 to $10 \mathrm{~dB}-$ Fig. 8. For values of SNR below $-5 \mathrm{~dB}$, it was not technically possible to clearly distinguish the 37 resolved peaks, with a fixed threshold.

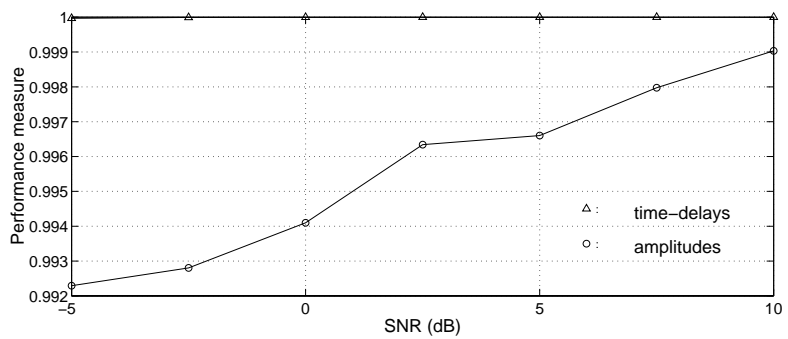

Fig. 8. Channel estimation performance in noise.

\section{CONCLUSIONS AND PERSPECTIVES}

This work has shown the feasibility of single sensor blind deconvolution by TF processing, for a realistic multiple time delay-attenuation channel, and a deterministic non-stationary source signal. The simulated data set was based on actual environmental data recorded at sea during a shallow water experiment, leading to a particularly difficult test case, where there are a high number of closely spaced multipaths. The deconvolved source signal showed a correlation with the emitted signal close to 0.8 , while the channel parameter estimation was shown to be effective and robust for SNR as low as $-5 \mathrm{~dB}$. Application of the proposed methods on at-sea recorded data is being performed at this time.

\section{REFERENCES}

[1] J. Ville, "Théorie et applications de la notion de signal analytique," Câbles et Transmissions, vol. 2A, pp. 61$74,1948$.

[2] R.G. Baraniuk and D.L. Jones, "A radially Gaussian, signal dependent time-frequency representation," in IEEE Int. Conf. Acoust., Speech and Sig. Proc., 1991, vol. May, pp. 3181-4.

[3] F. Hlawatsch and W. Krattenthaler, "Bilinear signal synthesis," IEEE Trans. Sig. Proc., vol. Feb., pp. 35263, 1992.

[4] P. Flandrin, "A time-frequency formulation of optimum detection," IEEE Trans. Acoust. Speech, Sig. Proc., vol. 36, No. 9, 1988.

[5] X. Démoulin, Y. Stéphan, S. Jesus, E. Coelho, and M.B. Porter, "Intimate96: a shallow water tomography experiment devoted to the study of internal tides," in Proc. SWAC'97, 1997.

[6] M. Porter, The Kraken Normal Mode Program, Saclant Undersea Research Centre, 1991.

[7] L. Cohen, Time-Frequency Analysis, Prentice Hall PTR, 1995.

[8] N.E. Martins, A time-frequency approach to blind deconvolution in multipath underwater channels, M.Sc. thesis, University of Algarve, 2001.

[9] C. Gervaise, A. Quinquis, and N. Martins, "Time-frequency approach to the study of underwater acoustic channel estimation and source reconstruction," in Physics in signal and image processing, Marseille, France, January 2001. 